\title{
Optical strain measurement for the modeling of surgical meshes and their porosity
}

Andreas Horbach, Biomechanics Laboratory, Institute for Bioengineering, FH Aachen University of Applied Sciences Heinrich-Mußmann-Str.1, Jülich, Germany, e-mail: horbach@fh-aachen.de

Manfred Staat, Biomechanics Laboratory, Institute for Bioengineering, FH Aachen University of Applied Sciences Heinrich-Mußmann-Str.1, Jülich, Germany, e-mail: staat@fh-aachen.de

The porosity of surgical meshes makes them flexible for large elastic deformation and establishes the healing conditions of good tissue ingrowth. The biomechanic modeling of orthotropic and compressible materials requires new materials models and simulstaneoaus fit of deformation in the load direction as well as trannsversely to to load. This nonlinear modeling can be achieved by an optical deformation measurement. At the same time the full field deformation measurement allows the dermination of the change of porosity with deformation. Also the socalled effective porosity, which has been defined to asses the tisssue interatcion with the mesh implants, can be determined from the global deformation of the surgical meshes. 


\section{Optimization of the cardiovascular stent design towards improved ex- pansion behaviour and radial stiffness properties}

Lisa Wiesent, Numerical Material Science Lab, Medical Device Lab, Regensburg Center of Biomedical Engineering, Ostbayerische Technische Hochschule Regensburg, lisa.wiesent@ oth-regensburg.de

Constantin Hupke, Medical Device Lab, Faculty of Mechanical Engineering, Ostbayerische Technische Hochschule Regensburg, constantin.hupke@st.oth-regensburg.de

Christian Balk, Medical Device Lab, Faculty of Mechanical Engineering, Ostbayerische Technische Hochschule Regensburg, christian.balk@st.oth-regensburg.de

Ulrich Schultheiß, Laboratory for Material Surface Layer Analytics, Faculty of Mechanical Engineering, Ostbayerische Technische Hochschule Regensburg, ulrich.schultheiss@oth-regensburg.de

Thomas Schratzenstaller, Medical Device Lab, Faculty of Mechanical Engineering, Regensburg Center of Biomedical Engineering, Ostbayerische Technische Hochschule Regensburg, thomas.schratzenstaller@oth-regensburg.de

Aida Nonn, Numerical Material Science Lab, Faculty of Mechanical Engineering, Ostbayerische Technische Hochschule Regensburg, aida.nonn@oth-regensburg.de

Coronary heart disease is one of the common cause of death in the industry countries. It is mainly treated by the implantation of a coronary stent. A major issue of stent treatment is in-stent restenosis, the re-narrowing of the vessel at the site of the implanted stent. In-stent restenosis is mainly related to vessel injuries during the expansion of the stent. Commonly stent expansion is initiated at the stent ends before advancing to the stent centre resulting in the usual dogbone shaped expansion behaviour. This leads to local stress concentrations and micro injuries of the vessel wall resulting in the proliferation of the vessel wall cells. To overcome this issue, stent expansion behaviour has to be improved by means of an expansion optimized stent design with reduced or in the best case with non-existing dogbone effect. In this work, the influence of stent design modification on its mechanical performance during the implementation has been investigated aiming at the reduction of vessel wall injuries. These investigations include numerical analysis of balloon folding, stent crimping and its free expansion with a folded balloon to quantify the effects of various stents designs on overall and local radial stiffness.. All numerical results are validated by experimental data such as highspeed recordings of stent expansion and collapse, scanning electron microscopy and embedded stents to ensure the realibility of developed approach. The obtained numerical and experimental results prove that already minor stent design modifications such as the increase of strut thickness at the stent ends can prevent the occurence of the undesired dogbone and increase the local and overall radial stiffness of the stent. All results still rely on the free stent expansion. The interaction of modified stents with the vessel wall will be investigated in future studies. 


\title{
Spatially Resolved Distensibility of Healthy, Diseased, and Aneurysmal Aortic Walls Determined from Temporally Resolved 3D Ultrasound Measurements
}

\author{
Andreas Wittek, Personalized Biomedical Engineering Laboratory, Frankfurt University of Applied Sciences, \\ Frankfurt am Main, Germany, wittek@fb2.fra-uas.de \\ Wojciech Derwich, Vascular and Endovascular Surgery, Goethe University Hospital, Frankfurt am Main, \\ Germany, Wojciech.Derwich@kgu.de \\ Thomas Schmitz-Rixen, Vascular and Endovascular Surgery, Goethe University Hospital, Frankfurt am Main, \\ Germany, schmitz-rixen@em.uni-frankfurt.de \\ Claus-Peter Fritzen, Institute of Mechanics and Control Engineering - Mechatronics, University of Siegen, \\ Germany, Claus-Peter.Fritzen@uni-siegen.de \\ Armin Huß, Personalized Biomedical Engineering Laboratory, Frankfurt University of Applied Sciences, \\ Frankfurt am Main, Germany, huss@fb2.fra-uas.de \\ Christopher Blase, Personalized Biomedical Engineering Laboratory, Frankfurt University of Applied Sciences, \\ Frankfurt am Main, Germany, cblase@fb2.fra-uas.de
}

\begin{abstract}
Abdominal aortic aneurysms (AAA) are a degenerative disease of the human aortic wall that may lead to weakening and eventually rupture of the wall with high mortality rates. Since the currently established criterion for surgical or endovascular treatment of the disease is imprecise in the individual case and treatment is not free of complications, the need for additional patient-individual biomarkers for short-term AAA rupture risk as basis for improved clinical decision making is widely acknowledged. Time resolved 3D ultrasound combined with speckle tracking algorithms is a novel non-invasive medical imaging technique that provides full-field displacement and strain measurements of aortic and aneurysmal wall motion. This is patient-individual information that has not been used so far to assess wall strength and rupture risk. In the current study we have used 4D ultrasound strain imaging to compute the spatially resolved distensibility distribution in three patient groups: young volunteers $<60 \mathrm{y}$. o. without known cardiovascular diseases, aged arteriosclerotic patients $>60 \mathrm{y}$. o. without AAA, and AAA patients. Local distensibilities were determined for wall areas of $2-10 \mathrm{~mm}^{2}$. Mean and maximum distensibilities as well as indices for the local variations of the distensibility distribution (local distensibility ratio, heterogeneity index) have been determined. Mean distensibility is significantly decreasing from young $\left(3.83[2.82,5.87] 10-3 \cdot \mathrm{mmHg}_{-1}\right)$ to elderly $(0.67[0.39,0.87] 10-3 \cdot \mathrm{mmHg}-1)$ and AAA $(0.27[0.20$, $0.54] 10-3 \cdot \mathrm{mmHg}-1)$. Both indices characterising the heterogeneous elastic properties are significantly increasing from young through elderly to AAA. We propose that the heterogeneous distribution is indicative of microstructural changes in the aortic wall. Therefore, the variable distribution of distensibilities might be a candidate biomarker to classify aneurysms with respect to their rupture risk.
\end{abstract}




\section{Numerical simulation of a transcatheter aortic heart valve under application-related loading}

Sylvia Pfensig, Institute for ImplantTechnology and Biomaterials e.V., Rostock-Warnemünde, Germany, sylvia.pfensig@uni-rostock.de

Sebastian Kaule, Institute for ImplantTechnology and Biomaterials e.V., Rostock-Warnemünde, Germany, sebastian.kaule@uni-rostock.de

Robert Ott, Institute for ImplantTechnology and Biomaterials e.V., Rostock-Warnemünde, Germany, robert.ott@uni-rostock.de

Carolin Wüstenhagen, Institute for ImplantTechnology and Biomaterials e.V., Rostock-Warnemünde, Germany, carolin.wuestenhagen@uni-rostock.de

Michael Stiehm, Institute for ImplantTechnology and Biomaterials e.V., Rostock-Warnemünde, Germany, michael.stiehm@uni-rostock.de

Jonas Keiler, Department of Anatomy, Rostock University Medical Center, Rostock, Germany, jonas.keiler@med.uni-rostock.de

Andreas Wree, Department of Anatomy, Rostock University Medical Center, Rostock, Germany, andreas.wree@med.uni-rostock.de

Niels Grabow, Institute for Biomedical Engineering, Rostock University Medical Center, Rostock-Warnemünde, Ger-many, niels.grabow@uni-rostock.de

Klaus-Peter Schmitz, Institute for ImplantTechnology and Biomaterials e.V. and Institute for Biomedical Engineering, Rostock University Medical Center, Rostock-Warnemünde, Germany, schmitz@iib-ev.de Stefan Siewert, Institute for ImplantTechnology and Biomaterials e.V., Rostock-Warnemünde, Germany, stefan.siewert@uni-rostock.de

For the treatment of severe symptomatic aortic valve stenosis, minimally invasive heart valve prostheses have more recently become the lifesaving solution for elderly patients with high operational risk and thus, are often implanted in patients with challenging aortic root configuration. A correct prosthesis deployment and stent adaption to the target region is essential to ensure optimal leaflet performance and long-term prosthesis function. The objective of this study was the development of a suitable in silico setup for structural numerical simulation of a transcatheter aortic valve (TAV) in different cases of clinical relevance. A transcatheter valve prosthesis comprising an unpressurized trileaflet heart valve and an adapted stent configuration was designed. An aortic root (AR) model was developed, based on microcomputed tomography of a native healthy specimen. Using the finite-element analysis (FEA), various loading cases including prosthesis biomechanics with valve opening and closing under physiological pressure ratios throughout a cardiac cycle, prosthesis crimping as well as crimping and release into the developed AR model were simulated. Hyperelastic constitutive law for polymeric leaflet material and super-elasticity of shape memory alloys for the selfexpanding Nitinol stent structure were implemented into the FEA setup. Calculated performance of the valve including the stent structure demonstrated enhanced leaflet opening and closing as a result of stent deformation and redirected loading. Crimping and subsequent release into the AR model as well as the stent adaption to the target region after expansion proved the suitability of the TAV design for percutaneous application. FEA represented a useful tool for numerical simulation of an entire minimally invasive heart valve prosthesis in relevant clinical scenarios. 


\title{
Time-harmonic elastography for the early detection of glomerulonephritis
}

\author{
Heiko Tzschätzsch, Department of Radiology Charité - Universitätsmedizin Berlin, Berlin, Germany, \\ heiko.tzschaetzsch@charite.de \\ Markus Grossmann, Department of Radiology Charité - Universitätsmedizin Berlin, Berlin, Germany, \\ markus.grossmann@charite.de \\ Stephan Rodrigo Marticorena Garcia, Department of Radiology Charité - Universitätsmedizin Berlin, Berlin, \\ Germany, stephan.marticorena-garcia@charite.de \\ Ingolf Sack, Department of Radiology Charité - Universitätsmedizin Berlin, Berlin, Germany, \\ ingolf.sack@charite.de
}

Glomerulonephritis has non-specific and subtle clinical signs. Beside laboratory tests and conventional ultrasound a kidney biopsy is required for a definitive diagnosis. Recently, time-harmonic elastography (THE), a non-invasive method to quantify tissue elasticity was introduced for the evaluation of renal stiffness in native liver and spleen. This novel ultrasound elastography method could be applied to detect structural changes in patients with glomerulonephritis.

THE, generates shear waves within the entire body by a patient bed with integrated loudspeaker. Propagating shear waves are captured over $1 \mathrm{~s}$ by a clinical B-mode ultrasound scanner. The acquired raw ultrasound data are send to an elastography PC which computes elastograms (shear wave speed (SWS) maps) within the entire fieldof-view up to $13 \mathrm{~cm}$ depth. For this, controlled aliasing, directional filtering and phase gradient wave number recovering are used.

In this study 37 healthy volunteers and 53 patients with glomerulonephritis underwent sonography as well as THE.

In all volunteers the cortex $(2.10 \mathrm{~m} / \mathrm{s})$ was stiffer compared to medullar $(1.35 \mathrm{~m} / \mathrm{s})$ similar to patients $(1.81 \mathrm{~m} / \mathrm{s}$ vs. $1.27 \mathrm{~m} / \mathrm{s})$. Strong correlation $(r=0.56, p=0.0002)$ was found between glomerular filtration rate $(\mathrm{GFR})$ and cortical SWS. The AUROC for the differentiation of healthy subjects from patients was $0.89,0.66$ and 0.54 for SWS, renal length and parenchyma thickness, respectively. Correspondingly, AUROCs were 0.82, 0.60 and 0.43 for the diagnosis of patients with normal GFR.

In conclusion, THE is able to distinguish cortical and medullar SWS. THE shows structural differences in patients with glomerulonephritis, as indicated by SWS changes, even in patients with normal renal function. Therefore, THE improves the early detection of glomerulonephritis and could potentially used for long-term monitoring. 


\section{Experimental setup for evaluation of cavitation effects in ESWL}

Nina Reinhardt, Chair of Medical Engineering, Helmholtz-Institute for Biomedical Engineering, RWTH Aachen University, Aachen, Germany, reinhardt@ hia.rwth-aachen.de

Kristin Dietz-Laursonn, Chair of Medical Engineering, Helmholtz-Institute for Biomedical Engineering, RWTH Aachen University, Aachen, Germany, dietzlaursonn@hia.rwth-aachen.de

Marc Janzen, Chair of Medical Engineering, Helmholtz-Institute for Biomedical Engineering, RWTH Aachen University, Aachen, Germany, janzen@hia.rwth-aachen.de

Christian Bach, University Hospital Aachen, Department of Urology, Aachen, Germany, chbach@ukaachen.de Klaus Radermacher, Chair of Medical Engineering, Helmholtz-Institute for Biomedical Engineering, RWTH Aachen University, Aachen, Germany, radermacher@ hia.rwth-aachen.de

Matias de la Fuente, Chair of Medical Engineering, Helmholtz-Institute for Biomedical Engineering, RWTH Aachen University, Aachen, Germany, fuente@hia.rwth-aachen.de

Cavitation is a major fracture mechanism in extracorporeal shock wave lithotripsy (ESWL). However, it can cause tis-sue trauma and its effects on kidney stones and surrounding tissue are not fully understood. Therefore experimental set-ups enabling systematic parameter studies are crucial. We developed and evaluated a testing rig comprising three measuring methods in order to examine this mechanism. Our initial evaluation of this setup based on standard components showed promising results. Primary cavitation was displayed by high-speed photography $195 \mu \mathrm{s}$ after the shock front had passed the focal zone. The effect of different pulse repetition rates $(30,60,90,120 \mathrm{SW} / \mathrm{min})$ on the extension of the cavitation area was determined. The lifetime of secondary cavitation was analysed by B-mode ultrasound imaging. In a post processing progress the images showing bubbles were compared to a reference picture for both types of cavitation and the number of pixels that changed colour was counted. Furthermore stone comminution at different pulse repetition rates (30, 60, 90, 120 SW/min) was investigated by fixed-dose fragmentation. We observed an inverse correlation of cavitation and fragmentation. As the pulse repetition rate increases, the area of primary cavitation grows whereas the fragmentation efficiency decreases. B-mode imaging showed that secondary cavitation bubbles persisted between the shocks and can serve as nuclei. The higher the pulse repetition rate is, the more of these nuclei remain and thus facilitate formation of primary cavitation. The experimental setup provides reproducible results regarding the development of primary and secondary cavitation on the one hand and the fragmentation of phantom stones on the other hand. Therefore it can be utilized to further investigate the effect of different boundary conditions and shock wave parameters on cavitation and stone comminution. The impact of different focal sound fields is subject of ongoing research. 


\section{Aortic regurgitation after transcatheter aortic valve replacement - Influence of valve prosthesis implantation depth inside the aortic annulus on paravalvular regurgitation in in vitro steady flow conditions}

Sebastian Kaule, Institute for ImplantTechnology and Biomaterials e.V., Rostock-Warnemünde, Germany Stefan Siewert, Sylvia Pfensig, Robert Ott, Carolin Wüstenhagen, Klaus-Peter Schmitz and Michael Stiehm, Institute for ImplantTechnology and Biomaterials e.V., Rostock-Warnemünde, Germany

Niels Grabow, Klaus-Peter Schmitz, Institute for Biomedical Engineering, Rostock University Medical Center, Rostock-Warnemünde, Germany

The assessment of hydrodynamic performance of transcatheter aortic valve prostheses in vitro is essential for the development and approval of novel devices. Therefore, this study aims to investigate the correlation of target implantation depth and paravalvular regurgitation in a controlled in vitro test situation. We designed a test setup with retrograde steady flow conditions measuring paravalvular regurgitation as a function of increasing pressure on the closed valve ranging from $0 \mathrm{mmHg}$ to $200 \mathrm{mmHg}$. Our future aim is to benchmark different valve prosthesis designs and describe the correlation between target implantation depth, paravalvular regurgitation and prosthesis design aspects.

The current study describes the developed test setup, validation experiments as well as first results for a selfexpanding valve prosthesis. The highest regurgitation was measured at an implantation depth of $2 \mathrm{~mm}$. In fact, regurgitation increases from $26.1 \pm 8.2 \mathrm{ml} / \mathrm{min}$ at $0 \mathrm{mmHg}$ to $1,490.7 \pm 182.7 \mathrm{ml} / \mathrm{min}$ at $160 \mathrm{mmHg}$. The slightest regurgitation, however, was measured for an implantation depth of $6 \mathrm{~mm}$ ranging from $2.2 \pm 0.6 \mathrm{ml} / \mathrm{min}$ at $0 \mathrm{mmHg}$ to $605.8 \pm 18.9 \mathrm{ml} / \mathrm{min}$ at $200 \mathrm{mmHg}$. 